\title{
Entanglement purification protocols for all graph states
}

\author{
Caroline Kruszynska. * A Akimasa Miyake, Hans J. Briegel, and Wolfgang Dür \\ Institut für Theoretische Physik, Universität Innsbruck, Technikerstr. 25, 6020 Innsbruck, Austria and \\ Institut für Quantenoptik und Quanteninformation der Österreichischen Akademie der Wissenschaften, Innsbruck, Austria
}

(Dated: June 9, 2006)

\begin{abstract}
We present multiparty entanglement purification protocols that are capable of purifying arbitrary graph states directly. We develop recurrence and breeding protocols and compare our methods with strategies based on bipartite entanglement purification in static and communication scenarios. We find that direct multiparty purification is of advantage with respect to achievable yields and minimal required fidelity in static scenarios, and with respect to obtainable fidelity in the case of noisy operations in both scenarios.
\end{abstract}

PACS numbers: 03.67.Mn, 03.67.Hk, 03.67.Pp

\section{INTRODUCTION}

Entanglement purification (distillation) is an important primitive in quantum information processing. It allows one to overcome the influence of noise in quantum communication [1, 2, 3], and enables one to obtain provable secure quantum key distribution even in the context of noisy channels [4] and over arbitrary distances [5]. The applications of entanglement purification are however not limited to bipartite communication scenarios. First, it has been extended to certain multiparty scenarios [6, 7, 8, 9, 10], leading e.g. to novel quantum primitives such as multiparty secure state distribution [11]. Second, in the context of fault-tolerant quantum computation, entanglement purification is a key ingredient to obtain improved error thresholds [12, 13]. Applications in quantum error correction [14] and quantum simulation 15] have also been discussed.

The basic idea of entanglement purification is to use several copies of a noisy entangled state to generate, by means of local operations and classical communication (LOCC), a few copies with improved fidelity. So far, entanglement purification protocols have been developed that are capable of purifying Bell states [1, 2, 3] in the bipartite case, and all two-colorable graph states [8, 9] as well as $\mathrm{W}$ states [16] in the multiparty case. They also have been generalized to higher dimensional systems [17]. Graph states [14, 18] are a family of multiparty entangled states with interesting entanglement properties. Two-colorable graph states are sub-family associated with two-colorable graphs and include a number of interesting states such as GHZ states, cluster states and codewords of CSS error correction codes. Graph states appear for instance in the context of measurement-based quantum computation, where a given graph state represents an algorithmic-specific resource that allows one to realize a specific unitary operation on several qubits by local measurements. Typically, these graphs are not two-colorable, but one may wish to purify this resource,

*Electronic address: caroline.kruszynski@uibk.ac.at e.g. to realize one-way quantum computation in a faulttolerant manner.

In this paper, we present entanglement purification protocols (EPP) that are capable of purifying arbitrary graph states. To be precise, we develop for each graph state a direct multiparty recurrence protocol and a multiparty breeding protocol. Our key ideas are twofold. First, we show that if auxiliary (even noisy) two-colorable graph states are available, the fidelity of the noisy graph states can be improved. Second, we show how to get a single copy of the auxiliary two-colorable graph state from two identical copies of the $k$-colorable graph state by LOCC. Thus, unlike the known two-colorable graph state entanglement purification protocol [8, 9] , our protocol utilizes different shapes of graphs.

The protocols are applicable both in (i) a static (LOCC) scenario, where the parties attempt to purify by LOCC several copies of given noisy multiparty entangled states, as well as in (ii) a communication scenario where the parties are allowed to generate (arbitrary) multiparty states locally, distribute them through noisy quantum channels and attempt to end up with high-fidelity target graph states. Our first idea is commonly utilized to both scenarios. The second idea is crucial in the static (LOCC) scenario, while in the communication scenario we may prepare separately auxiliary two-colorable graph states through noisy channels in an effective way.

In both cases, we show that the new direct multiparty EPP are superior to alternative approaches based on bipartite entanglement purification. In particular, we find that in the static scenario (i) the yield of direct multiparty breeding protocols is higher than for any strategy based on bipartite purification, and also the purification regime is larger. When also considering noisy local control operations, we show that for both scenarios (i) and (ii), the new multiparty recurrence protocol allows one to reach higher fidelities. We first review the concept of graph states in Sec. IIA, and then present new recurrence and breeding protocols in Sec. IV A and IV B. We present an alternative strategy based on purification of two-colorable sub graph-states in Sec. V. A comparison with bipartite strategies for noiseless in Sec. VIA and noisy local control operations in Sec.VIB finally demon- 
strates the advantage of these new protocols.

\section{GRAPH STATES AND MANIPULATION}

In this Section, we summarize the basics concerning graph states and their manipulations.

\section{A. Definition and notation for graph states}

Graph states are a family of multiparty entangled states associated with mathematical graphs [14]. A graph $G=(V, E)$ is given by a set $V=\{1,2, \ldots, N\}$ of $N$ vertices connected in a specific way by edges $E$. To every such graph there corresponds a basis of $N-$ qubit states $\left\{|\boldsymbol{\mu}\rangle_{G}\right\}$, where each of the basis states $|\boldsymbol{\mu}\rangle_{G}$ $\left(\boldsymbol{\mu}=\mu_{1} \mu_{2} \ldots \mu_{N}\right)$ is the common eigenstate of $N$ commuting correlation operators $K_{a}^{G}$ with eigenvalues $(-1)^{\mu_{a}}$ such that $\mu_{a}=0,1$. That is, they fulfill the set of eigenvalue equations $K_{a}^{G}|\boldsymbol{\mu}\rangle_{G}=(-1)^{\mu_{a}}|\boldsymbol{\mu}\rangle_{G}, a=1, \ldots, N$. The correlation operators are uniquely determined by the graph $G$ and are given by

$$
K_{a}^{G}=\sigma_{x}^{(a)} \prod_{\{a, b\} \in E} \sigma_{z}^{(b)}
$$

where $\sigma_{\alpha}^{(a)}$ denotes the application of the corresponding Pauli operator $(\alpha=x, y, z)$ by the party $a$. Equivalently, any graph state $|\boldsymbol{\mu}\rangle_{G}$ can be written in the following manner:

$$
|\boldsymbol{\mu}\rangle_{G}=\prod_{a=1}^{N}\left(\sigma_{z}^{(a)}\right)^{\mu_{a}}\left(\prod_{\{b, c\} \in E} \Lambda Z^{(b c)}\right)|+\rangle^{\otimes N}
$$

where $\Lambda Z=\operatorname{diag}(1,1,1,-1)$ in the computational basis is the controlled-phase gate, and $| \pm\rangle=\frac{1}{\sqrt{2}}(|0\rangle \pm|1\rangle)$.

We will use the concept of $k$-coloration in the following. A graph is called $k$-colorable if there exist $k$ sets of vertices $A_{1}, A_{2}, \ldots, A_{k} \in V$ such that there are no edges within each of the groups $A_{j}$ for all $j$, i.e. for all $a, b \in A_{j}$, and for all $j$, we have $\{a, b\} \notin E$. Two-colorable graphs are a special instance with $k=2$, where multiparty entanglement purification protocols are known [8, 9]. However, only a subset of graphs is two-colorable and, in principle, a graph may be $N$-colorable. We remark that local-unitary equivalent graph states [18] may correspond to graphs with different coloring, and the minimum $k$ within the local equivalence class is not known. Under local Clifford operation, we have however that generally $k>2$, i.e. not all graph states are locally equivalent to two-colorable graphs.

Associated with a given $k$-colorable graph $G$ with coloring $\left\{A_{1}, A_{2}, \ldots, A_{k}\right\}$, we define two-colorable graphs $\left\{g_{1}, g_{2}, \ldots, g_{k}\right\}$ (see Fig 1 for an illustration), where $g_{j}$ contains only the edges between the set $A_{j}$ and the remaining sets $\left\{A_{i}, i \neq j\right\}$, but where edges between the

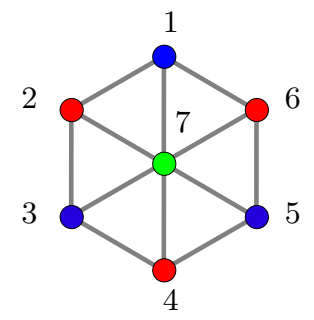

3-colorable graph $G$

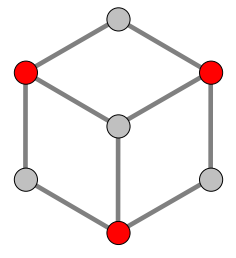

$g_{1}$

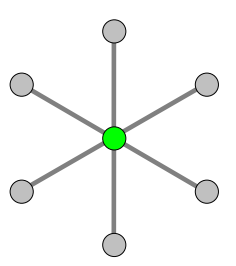

$g_{2}$

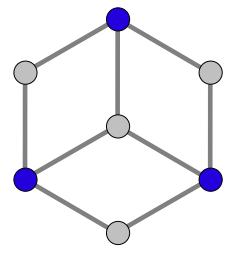

$g_{3}$
FIG. 1: (Color online). A 3-colorable graph $G$ and the 3 corresponding two-colorable sub-graphs $g_{1}, g_{2}$, and $g_{3} . g_{1}$ corresponds to the red color (vertices 2,4 and 6 ), $g_{2}$ to the green color (vertex 7 ) and $g_{3}$ to the blue (vertices 1,3 and 5 ).

remaining sets are erased. That is, the sets $\left\{A_{i}, i \neq j\right\}$ form a new set $A_{\bar{j}}=V \backslash A_{j}$. The set of indices $\mu_{a}$ corresponding to the set $A_{j}$ will be denoted by $\boldsymbol{\mu}_{j}$. Note that $\cup_{j=1}^{k} g_{j}=G$.

In this paper, we consider mixed states diagonal in the graph-state basis,

$$
\rho_{G}=\sum_{\boldsymbol{\mu}} \lambda_{\boldsymbol{\mu}_{1} \boldsymbol{\mu}_{2} \ldots \boldsymbol{\mu}_{k}}\left|\boldsymbol{\mu}_{1} \boldsymbol{\mu}_{2} \ldots \boldsymbol{\mu}_{k}\right\rangle_{G}\left\langle\boldsymbol{\mu}_{1} \boldsymbol{\mu}_{2} \ldots \boldsymbol{\mu}_{k}\right|,
$$

where we have grouped the multi-index $\boldsymbol{\mu}$ into $k$ multiindices $\boldsymbol{\mu}_{j}=\mu_{j_{1}} \ldots \mu_{j_{m}}\left(m=\left|A_{j}\right|\right)$ corresponding to the sets $A_{j}$ defined by a chosen $k$-coloration of the graph $G$, note that mixed states resulting from any noise models can be brought to this form by means of local depolarization, i.e. by applying randomly the local operations corresponding to the correlation operators $\left\{K_{a}^{G}\right\}$. Diagonal elements in the graph-state basis, i.e. the coefficients $\lambda_{\boldsymbol{\mu}_{1} \boldsymbol{\mu}_{2} \ldots \boldsymbol{\mu}_{k}}$ remain unchanged by this procedure, and any mixed state can be assumed to be of the form (3) without loss of generality. Hence we can interpret the mixed state as an ensemble of graph states $|\boldsymbol{\mu}\rangle_{G}$, where $|\boldsymbol{\mu}\rangle_{G}$ appears with probability $\lambda_{\boldsymbol{\mu}}$. We can therefore restrict our attention to the transfer of these indices between unknown pure states as presented in the next subsection.

\section{B. Operations on graph states}

We briefly mention two operations which play key roles in entanglement purification protocols. One is the multilateral CNOT which enables one to transfer the stabilizer eigenvalues $\boldsymbol{\mu}$ between two states. The other are Pauli 


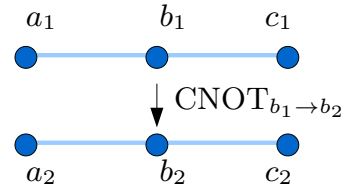

FIG. 2: (Color online). Effect of a CNOT gate on a product of two graph-states. The final state is associated to a graph composed of the two initial graphs with additional edges between the control qubit $b_{1}$ and all neighbors of the target qubit $b_{2}$.

measurements which allow one to evaluate $\boldsymbol{\mu}$ with the help of classical communication.

Let us first describe the action of a CNOT gate on a product of two (possibly different) $N$-qubit graph-states $|\boldsymbol{\mu}\rangle_{G_{1}}$ and $|\boldsymbol{\nu}\rangle_{G_{2}}$. We apply the CNOT gate from $a_{1}\left(a^{t h}\right.$ qubit of the first state) to $a_{2}$ ( $a^{\text {th }}$ qubit of the second state). We deduce the expression for the resulting state from (21). As $|+\rangle \otimes|+\rangle$ is an eigenvector of CNOT associated to the eigenvalue 1 , the difference between the initial and the final state is due to the commutation relation between the CNOT gate, the $\sigma_{z}$ operators and the controlled phase gates. A straightforward calculation yields

$$
\begin{aligned}
& \operatorname{CNOT}^{\left(a_{1} \rightarrow a_{2}\right)}|\boldsymbol{\mu}\rangle_{G_{1}}|\boldsymbol{\nu}\rangle_{G_{2}}= \\
& \quad\left(\sigma_{z}^{\left(a_{1}\right)}\right)^{\nu_{a_{2}}} \prod_{a_{2}^{\prime} \in N_{a_{2}}} \Lambda Z^{\left(a_{1} a_{2}^{\prime}\right)}|\boldsymbol{\mu}\rangle_{G_{1}}|\boldsymbol{\nu}\rangle_{G_{2}}
\end{aligned}
$$

where $N_{a_{2}}$ are the neighbors of vertex $a_{2}$. The final state is related to a graph of $2 N$ vertices which is composed of the two initial graphs with addition of all edges between vertex $a_{1}$ and the neighbors of vertex $a_{2}$. See Fig 2 for an illustration. In addition, bit $\mu_{a_{1}}$ is flipped if bit $\nu_{a_{2}}=1$, which corresponds to a transfer of information from the second state to the first one. Note however that this parity information cannot be evaluated without disturbing the first state.

To read out the information transfered between two states, one uses Pauli measurements in directions that correspond to the correlation operators $\left\{K_{a}^{G}\right\}$. Suppose we want to determine the bit string $\boldsymbol{\mu}_{j}$ corresponding to a color $A_{j}$ for a given graph state $\left|\boldsymbol{\mu}_{j}, \boldsymbol{\mu}_{\bar{j}}\right\rangle_{G}$. Then, the parties belonging to color $A_{j}$ measure their qubit $a$ in the eigenbasis $\left\{| \pm\rangle=\frac{1}{\sqrt{2}}(|0\rangle \pm|1\rangle)\right\}$ of $\sigma_{x}$, obtaining results $\xi_{a} \in\{0,1\}$, while all the other parties make their measurement in the eigenbasis $\{|0\rangle,|1\rangle\}$ of $\sigma_{z}$, obtaining results $\zeta_{b} \in\{0,1\}$. According to Eq. (1), each stabilizer eigenvalue $\mu_{a}$ of $K_{a}^{G}\left(a \in A_{j}\right)$ can be evaluated via classical communication of these measurement results as,

$$
\mu_{a}=\xi_{a} \bigoplus_{b \in N_{a}} \zeta_{b}
$$

Note that all indices corresponding to color $A_{j}$ are measured simultaneously.

\section{STATIC SCENARIO AND COMMUNICATION SCENARIO}

In this article, we are interested in the generation of high-fidelity graph states shared among $N$ spatially separated parties. We consider two cases (i) a static LOCC scenario, and (ii) a communication scenario, since both situations are of practical relevance in quantum information processing.

In (i), the static LOCC scenario, the parties share $M$ copies of a mixed state $\rho$. They can manipulate the states by means of LOCC in order to generate a high fidelity approximation of the target state $|\mathbf{0}\rangle_{G}$. No quantum communication between the parties is allowed. The efficiency of this procedure is measured by the yield, i.e. the ratio of high fidelity output state per input state. In (ii), the communication scenario, the parties are connected by noisy quantum channels and are allowed to distribute any locally generated entangled state through the noisy quantum channels, in addition to sequences of LOCC. The efficiency of this procedure is measured by the (inverse of the) quantum communication cost [19], i.e. the number of channel usages required to generate a desired multiparty entangled state with sufficiently high fidelity.

The scenario (i) deals with entanglement properties of a given mixed state $\rho$, i.e. whether high fidelity multiparty entangled states can be distilled from several copies of $\rho$ by LOCC. The scenario (ii), on the other hand, has a certain physical set-up (a communication set-up) in mind, and hence deals with the question whether in such a context high-fidelity entangled states can be generated. For our purposes, the most relevant difference between the two scenarios is that in case of (ii) noisy entangled states of any kind can be generated by distributing them through noisy quantum channels, which can be used to generate the desired target state. In (i), only copies of $\rho$ are given which should be manipulated by LOCC. If different states are needed, this has to be prepared by manipulating several copies of $\rho$ by LOCC.

\section{DIRECT MULTIPARTITE ENTANGLEMENT PURIFICATION PROTOCOL}

\section{A. Recurrence protocol}

We now present a recurrence protocol that allows one to purify directly any $k$-colorable graph-state, provided auxiliary two-colorable graph-states corresponding to the $k$ different colors are available. The sub-protocol is applicable in both scenarios, and we describe later on how to construct the auxiliary two-colorable graph-states in each scenario. Note that if the target graph state is twocolorable, auxiliary graphs $g_{1}$ and $g_{2}$ are nothing but $G$. Thus our protocol covers known protocols for twocolorable graph states. The total protocol consists of $k$ sub-protocols $\left\{\mathcal{P}_{j}\right\}(j=1, \ldots, k)$, each of which serves to purify a state partially, i.e. with respect to the index 
vector $\boldsymbol{\mu}_{j}$ corresponding to a group $A_{j}$ (i.e. a certain color). We outline the protocol in the following.

\section{Sub-protocol $\mathcal{P}_{j}$}

Assume that auxiliary (possibly noisy) two-colorable graph-states $\rho_{g_{j}}$ are available in addition to an ensemble of $k$-colorable graph-states $\rho_{G}$. We purify the $k$-colorable graph-states $\rho_{G}$ with respect to bit string $\boldsymbol{\mu}_{j}$ :

(1-1) The parties take two noisy states in such a way that the first state is $\rho_{G}$ and the second one is $\rho_{g_{j}}$. The parties in the group $A_{j}$ apply CNOT gates from the second (control) state $\rho_{g_{j}}$ to the first (target) state $\rho_{G}$, while the parties in the group $A_{\bar{j}}$ apply the CNOT gate in the opposite direction.

(1-2) The parties measure the second state $\rho_{g_{j}}$ locally in the eigenbasis of $\sigma_{x}$ for the group $A_{j}$ and in the eigenbasis of $\sigma_{z}$ for the group $A_{\bar{j}}$. Using classical communication, they decide to keep the first state $\rho_{G}$ if all eigenvalues corresponding to the correlation operators $\left\{K_{a}^{G}, a \in A_{j}\right\}$ of the group $A_{j}$ (determined by Eq. (5) ) are 0, or to discard it otherwise.

Let us take a close look at the sub-protocol $\mathcal{P}_{j}$. In (1-1), the multilateral CNOT operation,

$$
\prod_{\left\{a_{1}, a_{2}\right\} \in A_{j}} \mathrm{CNOT}^{\left(a_{2} \rightarrow a_{1}\right)} \prod_{\left\{b_{1}, b_{2}\right\} \in A_{\bar{j}}} \operatorname{CNOT}^{\left(b_{1} \rightarrow b_{2}\right)},
$$

is applied between a first state, corresponding to the graph $G$, and a second state corresponding to the graph $g_{j}$ (from now on, we use $a_{l}$ for the vertex of the $l$-th state in the group $A_{j}$ and $b_{l}$ for the one in the group $A_{\bar{j}}$ ). A straightforward calculation using Eq. (4) gives the following map

$$
\left|\boldsymbol{\mu}_{j}, \boldsymbol{\mu}_{\bar{j}}\right\rangle_{G}\left|\boldsymbol{\nu}_{j}, \boldsymbol{\nu}_{\bar{j}}\right\rangle_{g_{j}} \rightarrow\left|\boldsymbol{\mu}_{j}, \boldsymbol{\mu}_{\bar{j}} \oplus \boldsymbol{\nu}_{\bar{j}}\right\rangle_{G}\left|\boldsymbol{\nu}_{j} \oplus \boldsymbol{\mu}_{j}, \boldsymbol{\nu}_{\bar{j}}\right\rangle_{g_{j}},
$$

where $\oplus$ means bitwise addition modulo 2 , which shows the transfer of information about the stabilizer eigenvalues between the two states. Note that the final states correspond to the same graphs as the input states. After that, one measures the second state in order to determine the indices $\boldsymbol{\nu}_{j} \oplus \boldsymbol{\mu}_{j}$ corresponding to color $A_{j}$, using the procedure described in (1-2), where each bit $\nu_{j} \oplus \mu_{j}$ is determined using Eq. (5). If all the parities $\boldsymbol{\nu}_{j} \oplus \boldsymbol{\mu}_{j}$ are $\mathbf{0}$, it is expected that $\mu_{a}=0$ and $\nu_{a}=0$ for $a \in A_{j}$ are probable since $|\mathbf{0}\rangle_{G}$ and $|\mathbf{0}\rangle_{g_{j}}$ have been assumed to be the majority in their ensembles. That is why then the first state is kept and otherwise discarded. As consequence, in the expansion (3) of the density matrix, elements of the form $\lambda_{\mathbf{0}, \boldsymbol{\gamma}_{\bar{j}}}$ are increased. One finds that the new matrix elements of $\rho_{G}^{\prime}$ are given by

$$
\lambda_{\boldsymbol{\gamma}_{j}, \boldsymbol{\gamma}_{\bar{j}}}^{\prime}=\frac{1}{\kappa} \sum_{\left\{\left(\boldsymbol{\mu}_{\bar{j}}, \boldsymbol{\nu}_{\bar{j}}\right) \mid \boldsymbol{\mu}_{\bar{j}} \oplus \boldsymbol{\nu}_{\bar{j}}=\boldsymbol{\gamma}_{\bar{j}}\right\}} \lambda_{\boldsymbol{\gamma}_{j}, \boldsymbol{\mu}_{\bar{j}}} \tilde{\lambda}_{\boldsymbol{\gamma}_{j}, \boldsymbol{\nu}_{\bar{j}}}
$$

where $\kappa$ is a normalization constant guaranteeing that $\operatorname{tr}\left(\rho^{\prime}\right)=1$, and $\tilde{\lambda}$ are the coefficients for the two-colorable graph-state $\rho_{g_{j}}$ written in the form (3). We remark that here we do not address the question of the unfavorable scaling behavior of the efficiency of the proposed protocol with the total number of particles $N$, for recent developments on this subject in the context of two-colorable graph state purification see [20].

We illustrate how the protocol works by looking at the simple toy-case where noise acts only on one color. Let us consider $G$ as the 5 -qubit ring and $g_{1}$ as the 5 -qubit cluster, and study the effect of $\mathcal{P}_{1}$ on mixed states of the form

$$
\begin{aligned}
\rho_{G} & =\sum_{\boldsymbol{\mu}_{1}} \lambda_{\boldsymbol{\mu}_{1}, \mathbf{0}, 0}\left|\boldsymbol{\mu}_{1}, \mathbf{0}, 0\right\rangle_{G}\left\langle\boldsymbol{\mu}_{1}, \mathbf{0}, 0\right|, \\
\rho_{g_{1}} & =\sum_{\boldsymbol{\nu}_{1}} \tilde{\lambda}_{\boldsymbol{\nu}_{1}, \mathbf{0}, 0}\left|\boldsymbol{\nu}_{1}, \mathbf{0}, 0\right\rangle_{g_{1}}\left\langle\boldsymbol{\nu}_{1}, \mathbf{0}, 0\right| .
\end{aligned}
$$

Note that even though $g_{1}$ is two-colorable, we group the vertices in three distinct sets corresponding to the colors of the ring. As only the qubits in $A_{1}$ are noisy, a sequence of applications of sub-protocol $\mathcal{P}_{1}$ is sufficient to purify the state. A straightforward calculation gives the new coefficient of the purified ring-state: $\lambda_{\boldsymbol{\gamma}_{1}, \mathbf{0}, 0}^{\prime}=\lambda_{\boldsymbol{\gamma}_{\mathbf{1}}, \mathbf{0}, 0} \tilde{\lambda}_{\boldsymbol{\gamma}_{\mathbf{1}}, \mathbf{0}, 0} / \sum_{\boldsymbol{\nu}_{1}}\left(\lambda_{\boldsymbol{\nu}_{\mathbf{1}}, \mathbf{0}, 0} \tilde{\lambda}_{\boldsymbol{\nu}_{\mathbf{1}}, \mathbf{0}, 0}\right)$, meaning that the dominant coefficients are increased.

The whole purification protocol consists of a sequence of applications of the sub-protocols $\mathcal{P}_{j}$ corresponding to all colors $j=1, \ldots, k$. Even though there is a back-action of noise for the colors which are not purified for the step $j$, one obtains an overall increase of the fidelity $\lambda_{\mathbf{0}}$ if the fidelity of the initial state is sufficiently high. In fact, $\lambda_{\mathbf{0}}=1$ is an attractive fix point of the protocol under the ideal local operations.

\section{Preparation of the auxiliary state $\rho_{g_{j}}$ in static scenario}

Next we describe how to obtain the auxiliary twocolorable graph-states $\rho_{g_{j}}$ required for sub-protocol $\mathcal{P}_{j}$. In the communication scenario, these states can be generated directly by distributing them through (noisy) quantum channels. In the static scenario, the situation is slightly more complicated and a pre-processing to the purification sub-protocol $\mathcal{P}_{j}$ is needed which we will describe in the following.

Assume that an ensemble of noisy $k$-colorable graph states $\rho_{G}$ is available. We supply an ensemble of the auxiliary two-colorable graph state $\rho_{g_{j}}$ by LOCC:

(0-1) The parties take two identical noisy copies of $\rho_{G}$. The parties in the group $A_{j}$ apply a CNOT gate from the second (control) copy to the first (target) copy, the parties in the group $A_{\bar{j}}$ apply the CNOT in the opposite direction. 
(0-2) The parties measure the second state in the eigenbasis of $\sigma_{z}$. By this, they erase all the edges between the two states and they are left with a state corresponding to the two-colorable graph $g_{j}$ after a suitable change of local basis depending on the measurement outcome.

A straightforward calculation using Eq. (41) shows that the multilateral CNOT applied in step (0-1) results in the following map when applied to a product of two $k$ colorable graph-states

$$
\begin{aligned}
& \left|\boldsymbol{\mu}_{j}, \boldsymbol{\mu}_{\bar{j}}\right\rangle_{G}\left|\boldsymbol{\nu}_{j}, \boldsymbol{\nu}_{\bar{j}}\right\rangle_{G} \\
\mapsto & \prod_{\substack{b_{1} \in A_{\bar{j}} \\
b_{2}^{\prime} \in N_{b_{2}} \cap A_{\bar{j}}}} \Lambda Z^{\left(b_{1} b_{2}^{\prime}\right)}\left|\boldsymbol{\mu}_{j}, \boldsymbol{\mu}_{\bar{j}} \oplus \boldsymbol{\nu}_{\bar{j}}\right\rangle_{g_{j}}\left|\boldsymbol{\nu}_{j} \oplus \boldsymbol{\mu}_{j}, \boldsymbol{\nu}_{\bar{j}}\right\rangle_{G},
\end{aligned}
$$

which is a product of a graph-state associated to $g_{j}$ (state 1 ) and a graph-state associated to $G$ (state 2 ), with additional edges between the two graphs. The local $\sigma_{z}$ measurements on the second state (step (0-2)) erase the corresponding vertices and all edges associated to them in the graph. This ensures that after the measurement the first state is a two-colorable graph state corresponding to the graph $g_{j}$ as desired.

Note that the multilateral CNOT does not only create the desired two-colorable graph-state, it also results in a transfer of information between the two states. In particular, the part of the index bit of state 2 corresponding to color $A_{j}$ is given by $\boldsymbol{\nu}_{j} \oplus \boldsymbol{\mu}_{j}$, where $\boldsymbol{\mu}$ and $\boldsymbol{\nu}$ correspond to the states before the CNOT operation. We are therefore not only able to create the two-colorable graphstate but also to perform at the same time a first step of purification. To this aim, we replace the measurement in the eigenbasis of $\sigma_{z}$ by a measurement of the correlation operators $K_{a}^{G}$ with $a \in A_{j}$, where we keep the state only if the expectation values of all these correlation operators are zero. That is, step (0-2) can be replaced by

(0-2)' The parties measure the second state locally in the eigenbasis of $\sigma_{x}$ for the group $A_{j}$ and in the eigenbasis of $\sigma_{z}$ for the group $A_{\bar{j}}$. The first state is kept if all eigenvalues corresponding to correlation operators $\left\{K_{a}^{G}, a \in A_{j}\right\}$ of the group $A_{j}$ (determined by Eq. (5) ) are 0, and discarded otherwise.

Note that depending on the measurement outcomes a local unitary operation on the remaining copy is required to ensure that $|\mathbf{0}\rangle_{g_{i}}$ is the dominant component of the resulting state $\rho_{g_{j}}$. To be precise, the parties should apply a local unitary operation $\prod_{b_{1} \in A_{\bar{j}}, b_{2}^{\prime} \in N_{b_{2}} \cap A_{\bar{j}}}\left(\sigma_{z}^{\left(b_{1}\right)}\right)^{\zeta_{b_{2}^{\prime}}}$ depending on their measurement pattern $\zeta_{b_{2}^{\prime}}$ after either $(0-2)$ or $(0-2)^{\prime}$.

\section{B. Breeding and hashing protocols}

Hashing and breeding protocols were introduced for the bipartite Bell state in Ref. [1, 2], for the GHZ state in
Ref. 7], and generalized to all two-colorable graph states in Ref. 8,4 , 10, 21]. We will now show that the multilateral CNOT operation together with the use of different states allow one to construct hashing and breeding protocols for any graph state. In both cases, we are given an ensemble of $M$ imperfect $k$-colorable graph-states, with $M \rightarrow \infty$. One then transfers information from randomly chosen subsets of $m$ states to perfect states (breeding) or imperfect states (hashing), which are then measured. At each round our knowledge about the remaining states is increased.

We propose here a generalization of the breeding protocol to all graph-states. A hashing protocol can be obtained in a similar manner with the additional requirement of taking into account the back-action due to the imperfection of the states used to read out the information. One is given $M$ copies of an $N$-qubit graph-state corresponding to graph $G$, where $G$ is $k$-colorable. We consider $M \rightarrow \infty$. In addition, one is also given $k$ ensembles $E_{j}, j=1, \ldots, k$, which one needs to give back at the end, where ensemble $E_{j}$ contains perfect copies of the two-colorable graph-state corresponding to graph $g_{j}$. Let us call $B_{i}, i \in 1, \ldots, N$ the binary vector which contains the value of bit $i$ for a sequence of $m$ states. It is possible to determine the parity of all $B_{i}$ belonging to a given colour $A_{j}$ simultaneously by measuring only one state. This is done by performing CNOT gates from one state of $E_{j}$ to the $m k$-colorable graph states for all qubits in $A_{j}$ and in the opposite direction for the other qubits. To recover the parity of $B_{i}$, one measures the two-colorable graph-state using the method described in Seq. IIB, By this, one determines the eigenvalues of all correlation operators corresponding to set $A_{j}$, which are given by (5). One needs to repeat the procedure at most $M S\left(a_{i}^{(0)}, a_{i}^{(1)}\right)$ times to obtain perfect knowledge of $B_{i}$, where $S\left(a_{i}^{(0)}, a_{i}^{(1)}\right)=-a_{i}^{(0)} \log _{2} a_{i}^{(0)}-a_{i}^{(1)} \log _{2} a_{i}^{(1)}$ is the entropy and $a_{i}^{\left(\mu_{i}\right)}=\sum_{\mu_{k} \neq \mu_{i}} \lambda_{\mu_{1} \mu_{2} \ldots \mu_{i} \ldots \mu_{N}}$.

For each of the $k$ colors, one performs the sequence of operations described above $\max _{i \in A_{j}}\left(M S\left(a_{i}^{(0)}, a_{i}^{(1)}\right)\right)$ times in order to obtain the parity of all $B_{i}$ belonging to this color. Given this information, one ends up with a pure state corresponding (up to local unitary operations) to $M^{\prime}$ copies of $|\mathbf{0}\rangle_{G}$. The last step consists in reconstituting the pool of perfect two-colorable graph-states we were given at the beginning. This is done by performing the multilateral CNOT given by (6) on two copies, using by this $2 \max _{i \in A_{j}}\left(M S\left(a_{i}^{(0)}, a_{i}^{(1)}\right)\right) k$-colorable graph-states for each color. A lower bound $Y$ of the yield is therefore given by

$$
Y=1-2 \sum_{j=1}^{k} \max _{i \in A_{j}} S\left(a_{i}\right) .
$$

We remark that this construction might not be optimal, and a significant improvement of the yield could be achieved by an optimal procedure that generates $M^{\prime}$ copies $\left|\Psi_{g_{j}}\right\rangle$ from $M$ copies of $\left|\Psi_{G}\right\rangle$. To illustrate our 


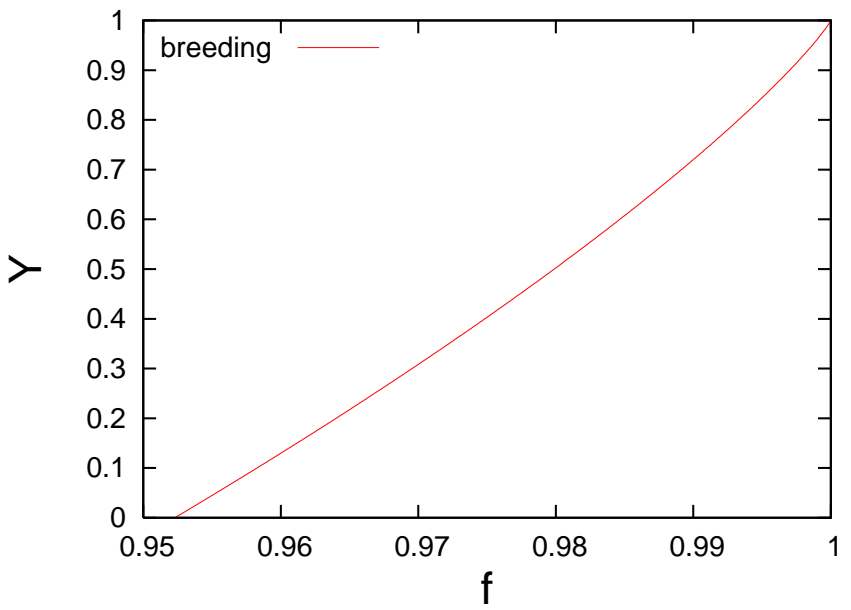

FIG. 3: Yield as function of fidelity for the breeding protocol applied to the 5-qubit ring-state.

protocol, we calculated the yield for a 5-qubit ring state of the form

$$
\rho=f|\mathbf{0}\rangle_{G}\langle\mathbf{0}|+(1-f) /\left(2^{5}-1\right)\left(\mathbb{1}-|\mathbf{0}\rangle_{G}\langle\mathbf{0}|\right) .
$$

Note that this state is a 3 -colorable graph-state. The entropy $S\left(a_{i}^{(0)}, a_{i}^{(1)}\right)$ is identical for all bits. It is given by

$$
\begin{aligned}
& S\left(a_{1}^{(0)}, a_{1}^{(1)}\right)= \\
& -\left(f+\left(2^{4}-1\right) \frac{1-f}{2^{5}-1}\right) \log _{2}\left(f+\left(2^{4}-1\right) \frac{1-f}{2^{5}-1}\right) \\
& \quad-\left(2^{4} \frac{1-f}{2^{5}-1}\right) \log _{2}\left(2^{4} \frac{1-f}{2^{5}-1}\right)
\end{aligned}
$$

The yield, given by $\left(1-6 S\left(a_{1}^{(0)}, a_{1}^{(1)}\right)\right)$ is plotted as function of $f$ in Fig. 3. One sees that the yield is approaching 1 for a state of fidelity close to 1 .

\section{ALTERNATIVE PURIFICATION PROTOCOLS VIA CUTTING INTO AND RECONNECTING 2-COLORABLE GRAPH STATES}

Until now we have considered strategies where the $k$ colorable graph-state was directly purified. Another possibility is to purify smaller parts of the graph-state corresponding to two-colorable sub-graphs and connect them at the end. The advantage of this strategy is to make possible the usage of known two-colorable graph state purification protocols. Different intermediate strategies can be designed depending on the chosen sub-graphs. We use an iterative method to construct a set of sub-graphs with no overlapping edges, such that their union is the graph $G$. Step $j$ of the procedure consists of choosing color $A_{j}$ out of the $k-j$ remaining colors and constructing sub-graph $\tilde{g_{j}}$, such that $\tilde{g_{j}}$ is a further sub-graph $g_{j}$ of Sec. IIA where all vertices $a \in A_{i}, i=1, \ldots, j-1$ have been
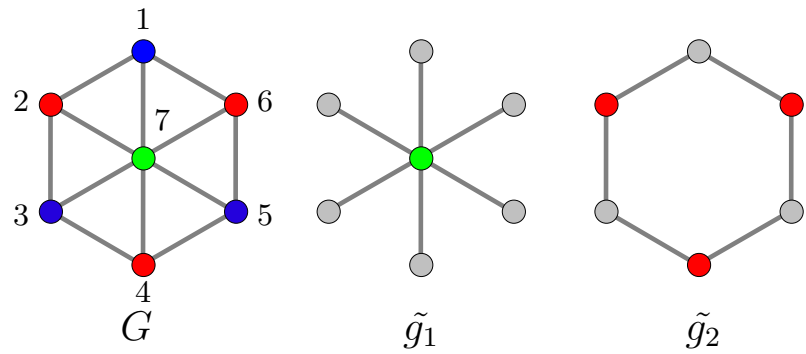

FIG. 4: (Color online). A 3-colorable graph $G$ with colors $A_{1}=\{7\}, A_{2}=\{2,4,6\}$ and $A_{3}=\{1,3,5\} . \quad \tilde{g_{1}}$ and $\tilde{g_{2}}$ are two-colorable sub-graph which can be obtained from $G$ and which give $G$ when merging them together. We use the method described in Sec. $\nabla$ to construct the sub graphs. For example, to obtain $\tilde{g_{1}}$, we choose $A_{1}$ as first color and erase all edges between $A_{2}$ and $A_{3}$ using the procedure described in Sec. IVA2 The sub-graph $\tilde{g_{2}}$ is obtained by erasing the vertex $A_{1}$ via a $\sigma_{z}$ measurement, and no further processing is required since this graph is already 2 -colorable.

erased. At each step, one checks if the graph $G \backslash\left\{\cup_{i=1}^{j} \tilde{g}_{i}\right\}$ is two-colorable. If this is the case, one stops the procedure. The states corresponding to the sub-graphs are distributed from the beginning in a communication scenario, while they are generated from two copies using the multilateral CNOT operation described in Sec. IVA in a static scenario, followed by a measurement in the $\sigma_{z}$ basis of the vertices one wants to erase. Once the states are distributed, the multipartite purification protocol for twocolorable graph-states [8, 9] is applied. To conclude the protocol, one merges the different graph states together in order to create the final $k$-colorable graph-state. Given two vertices $a_{1}$ and $a_{2}$ belonging to different graphs, the corresponding party merges them by applying a projective measurement given by $P_{0}=|0\rangle\langle 00|+| 1\rangle\langle 11|$ and $P_{1}=|0\rangle\langle 01|+| 1\rangle\langle 10|$ (with outcomes 0 and 1 ). A local correction given by $\prod_{b \in N_{a_{2}}} \sigma_{z}^{(b)}$ is applied to the state when the measurement result is 1 .

\section{COMPARISON WITH BIPARTITE STRATEGIES}

\section{A. Performance under ideal local operations in static scenario}

\section{Yield}

We show in this section that in a static scenario, direct multipartite purification is more efficient than strategies based on bipartite purification. This is due to the fact that any strategy based on bipartite entanglement purification requires that at a certain stage Bell-pairs shared among pairs of parties are generated. After the entanglement purification protocol, another sequence of LOCC must be applied to recover the multiparty entan- 
gled states. We show here that these two sequences of operations necessarily generate losses even when applied to pure states. We illustrate this by considering as example the 5-qubit ring state to which we apply the method introduced in Ref. [9] to quantify the loss. We start with an ensemble of $M$ perfect ring states $|\mathbf{0}\rangle_{G}\langle\mathbf{0}|$, which are then transformed to an ensemble of Bell pairs $|\mathbf{0}\rangle_{G_{2}}^{(k l)}\langle\mathbf{0}|$, shared between the different parties, by means of LOCC. Another sequence of LOCC brings the pairs to an ensemble of $\tilde{M}$ ring states. The total procedure can be summarized as follows:

$$
|\mathbf{0}\rangle_{G}\left\langle\left.\mathbf{0}\right|^{\otimes M} \rightarrow \bigotimes_{(k, l) ; k<l} \mid \mathbf{0}\right\rangle_{G_{2}}^{(k l)}\left\langle\left.\mathbf{0}\right|^{\otimes m_{k l}} \rightarrow \mid \mathbf{0}\right\rangle_{G}\left\langle\left.\mathbf{0}\right|^{\otimes \tilde{M}}\right.
$$

We now calculate a bound on the yield $\tilde{M} / M$ for this procedure. To do it, we apply the following inequalities which were used in Ref. 23] to show the irreversibility of entanglement transformation between singlets and GHZ states: (a) The entropy can only decrease on average under LOCC operations; (b) The average increase of relative entropy of system $B=V \backslash A$ is smaller than the average decrease of entropy in system $A$. If we consider a density operator $\rho_{A B}$ describing a pure state which is transformed to an ensemble $\left\{p_{k}, \tilde{\rho}_{A B}^{(k)}\right\}$ by LOCC operations we have that (a)

$$
S\left(\rho^{A}\right) \geq \sum_{k} p_{k} S\left(\tilde{\rho}_{k}^{A}\right)
$$

where $S\left(\rho^{A}\right)$ is the von Neumann entropy of the reduced density operator for system $A, \rho$ is the initial state and $\tilde{\rho}$ the final state. We consider the bipartition of the system of qubits into system $A$ and system $B=V \backslash A$. Then, we have (b)

$$
\sum_{k} p_{k} E_{r}\left(\tilde{\rho}_{k}^{B}\right)-E_{r}\left(\rho^{B}\right) \leq S\left(\rho^{A}\right)-\sum_{k} p_{k} S\left(\tilde{\rho}_{k}^{A}\right),
$$

where $E_{r}\left(\rho^{A}\right)$ is the relative entropy of entanglement for $\rho^{A}$

$$
E_{r}\left(\rho^{A}\right)=\min _{\sigma^{A} \text { separable }} S\left(\rho^{A} \| \sigma^{A}\right)
$$

with

$$
S\left(\rho^{A} \| \sigma^{A}\right):=\operatorname{Tr}\left(\rho^{A} \log \rho^{A}\right)-\operatorname{Tr}\left(\rho^{A} \log \sigma^{A}\right)
$$

being the relative entropy. Let us now apply this inequalities to our example. We first calculate (16) for the second part of process (15) with $A=\left\{a_{1}, a_{2}\right\}$ and $B=V \backslash A$. We have for the state describing the ensemble of pairs $S\left(\rho^{A}\right)=\sum_{b ; b \in B}\left(m_{a_{1} b}+m_{a_{2} b}\right)$. This comes from the fact that the entropy of a pure state is zero and that $\operatorname{Tr}_{B} \rho$ is not a pure state if and only if a single of the 2 qubits belonging to set $A$ is part of an entangled pair. In this case, the entropy is equal to unity for a single state. As there are $m_{a b}$ states containing a fully entangled pair between qubits $a$ and $b$, the contribution of this states to the total entropy is $m_{a b}$. For the $\tilde{M}$ ring states we find $S\left(\tilde{\rho}_{A}\right)=2 \tilde{M}$, as $\operatorname{Tr}_{B}\left(|\mathbf{0}\rangle_{G}\langle\mathbf{0}|\right)$ can be written as a sum of 4 projectors with equal weights (see below), and hence for a single copy of the ring state, the entropy of entanglement with respect to the bipartition in question is two. Summing up the contributions of all bipartitions of 2 and 3 qubits, we obtain

$$
6 \sum_{a, b ; a<b} m_{a b} \geq 20 \tilde{M}
$$

We now apply inequality (17) to the first part of process (15), with $A=\left\{a_{1}, a_{2}\right\}$ and $B=V \backslash A$, to have a bound on $M$. We distinguish two kinds of bipartitions: (i) qubits $a_{1}$ and $a_{2}$ are neighbors (ii) they are not neighbors. Let us study the entanglement properties of the state $|\mathbf{0}\rangle_{G}\langle\mathbf{0}|$ obtained by tracing out $A$. In case (i), $\operatorname{Tr}_{A}\left(|\mathbf{0}\rangle_{G}\langle\mathbf{0}|\right)=1 / 4\left(|000\rangle_{G^{\prime}}\langle 000|+\right.$ $\left.|001\rangle_{G^{\prime}}\langle 001|+| 100\rangle_{G^{\prime}}\langle 100|+| 101\rangle_{G^{\prime}}\langle 101|\right)$, where $G^{\prime}$ stands for the graph corresponding to the 3-qubit GHZ state. A straightforward calculation shows that this state is separable as it can be written as $\operatorname{Tr}_{A}\left(|\mathbf{0}\rangle_{G}\langle\mathbf{0}|\right)=$ $(\operatorname{Had} \otimes \mathbb{1} \otimes \operatorname{Had})^{\dagger} \rho^{\prime}(\operatorname{Had} \otimes \mathbb{1} \otimes \operatorname{Had})$, with $\rho^{\prime}=1 / 4(\mid+$ $++\rangle\langle+++|+|--+\rangle\langle--+|+|-+-\rangle\langle-+$ $-|+|+--\rangle\langle+--|)$. Similarly in case (ii) we find $1 / 4\left(\mathbb{1} \otimes\left(\mathbb{1}+\mathrm{i} \sigma_{\mathrm{z}}\right) \otimes\left(\mathbb{1}+\mathrm{i} \sigma_{\mathrm{z}}\right)\right)^{\dagger} \operatorname{Tr}_{A}\left(|\mathbf{0}\rangle_{G}\langle\mathbf{0}|\right)(\mathbb{1} \otimes$ $\left.\left(\mathbb{1}+\mathrm{i} \sigma_{\mathrm{z}}\right) \otimes\left(\mathbb{1}+\mathrm{i} \sigma_{\mathrm{z}}\right)\right)=(\mathbb{1} \otimes \operatorname{Had} \otimes \operatorname{Had})^{\dagger} \rho^{\prime}(\mathbb{1} \otimes$ $\mathrm{Had} \otimes \mathrm{Had})$. Hence the state obtained after tracing out 2 qubits is always separable, which implies that the relative entropy $E_{r}$ vanishes. In addition, we have $S\left(\rho_{A}\right)=S\left(\rho_{B}\right)$ and using the decomposition above we obtain $S\left(\operatorname{Tr}_{B}\left(|\mathbf{0}\rangle_{G}\langle\mathbf{0}|\right)\right)=2$. The relative entropy of entanglement of the ensemble of pairs is given by $E_{r}\left(\tilde{\rho}^{B}\right)=\sum_{\{b, c\} \in B} m_{b c}$ as $E_{r}=1$ for a fully entangled pair and $E_{r}=0$ for a separable state. We sum up the contributions of the $\left(\begin{array}{l}5 \\ 2\end{array}\right)$ possible bipartitions to obtain

$$
9 \sum_{a, b ; a<b} m_{a b} \leq 20 M
$$

Joining the two inequalities we get

$$
\tilde{M} \leq \frac{2}{3} M
$$

and hence the procedure (15) generates losses. On the other hand, breeding presented in Sec. IVB (or equivalently hashing) gives yield 1 for states of fidelity 1 , and for a state of form (13) with $f>0.9877$ we have $Y>2 / 3$. Hence, any 5-qubit ring-state arising from the application of global depolarizing noise and with fidelity $f>0.9877$, can be purified more efficiently using the direct protocol.

\section{Minimal required fidelity}

In this section, we illustrate the process consisting in generating one entangled pair from a 5-qubit ring-state. We see that the minimal required fidelity is higher for 
the bipartite strategy. We start with a state resulting from the application of global white noise to the 5-qubit ring-state, given by Eq. 13 . We define $x=f-\frac{1-f}{2^{5}-1}$ and rewrite the state as

$$
\rho=x|\mathbf{0}\rangle_{G}\langle\mathbf{0}|+\frac{(1-x)}{2^{5}} \mathbb{1},
$$

where $G$ stands for the ring. To create a 2 -qubit entangled pair from the initial state, we measure three consecutive qubits in the eigenbasis of $\sigma_{z}$. We remark that this is in fact an optimal strategy for states of the form Eq. (23) when operating on a single copy. The resulting state of the remaining two qubits, after some local correction if the measurement result is 1 , is given by

$$
\rho=x|\mathbf{0}\rangle_{G_{2}}\langle\mathbf{0}|+\frac{(1-x)}{2^{2}} \mathbb{1},
$$

where $G_{2}$ is the graph consisting in two vertices connected by an edges. This state is equivalent up to local unitary operations to a Werner state with fidelity $F=(3 x+1) / 4$. For $x>1 / 3$ the state is distillable since bipartite entanglement purification protocols can be successfully applied. The state has positive partial transpose for $x \leq 1 / 3$ which implies that it is not distillable. We thus have the condition $x>1 / 3$ such that the state is distillable via a bipartite entanglement purification strategy. For the direct multiparty entanglement purification protocol proposed in this article, we (numerically) find a threshold $x>0.2$ for states of the form Eq. (23). Thus the minimum required fidelity for the multiparty strategy is significantly lower than for the bipartite strategy. Although here we illustrate the advantage of our method by the 5 -qubit ring state, such advantages are expected for other graph states as well.

\section{B. Performance under imperfect local operations}

After having shown the advantage of multipartite purification with respect to bipartite purification in the static scenario, we turn to a more general setting including noisy local operations. We show here that when local operations are imperfect, multipartite purification can be advantageous also in the communication scenario.

We model noise in the communication channels and in the local operations as follows. We study typical noise models, where the Kraus representation of the superoperators is diagonal in the Pauli basis. This is a common and usually sufficiently general model 22] (in particular, any noisy channel can be brought to such a form by means of (probabilistic) local operations). We consider the depolarizing channel:

$$
\begin{aligned}
& \mathcal{E}_{p}^{(a)}(\rho)=p \rho+\frac{1-p}{4}\left(\rho+\sigma_{x}^{(a)} \rho \sigma_{x}^{(a)}+\right. \\
&\left.\sigma_{y}^{(a)} \rho \sigma_{y}^{(a)}+\sigma_{z}^{(a)} \rho \sigma_{z}^{(a)}\right)
\end{aligned}
$$

where $p$ is the reliability. As part of the purification protocols, local one- and two-qubit unitary operations are employed which may be noisy. An imperfect operation is modeled by preceding the perfect operation $U^{\left(a_{1} a_{2}\right)}$ with the application of the noise superoperators $\mathcal{E}$ from Eq. (25) with parameter $p_{l}$, i. e. the state is transformed as

$$
\rho \mapsto U^{\left(a_{1} a_{2}\right)}\left(\mathcal{E}_{p_{l}}^{\left(a_{1}\right)} \mathcal{E}_{p_{l}}^{\left(a_{2}\right)}(\rho)\right) U^{\dagger\left(a_{1} a_{2}\right)} .
$$

We assume that the protocols are executed with the least possible number of operations to keep accumulated noise low. Hence, if a local two-qubit gate $U_{12}^{\left(a_{1} a_{2}\right)}$ is preceded by one-qubit gates $U_{1}^{\left(a_{1}\right)}$ and $U_{2}^{\left(a_{2}\right)}$ we apply one combined unitary $U^{\left(a_{1} a_{2}\right)}=U_{1}^{\left(a_{1}\right)} U_{2}^{\left(a_{2}\right)} U_{12}^{\left(a_{1} a_{2}\right)}$ which is subjected to noise only once.

We compare a strategy using direct multiparty entanglement purification (MEPP strategy) with a strategy using bipartite entanglement purification (BEPP strategy) in the particular example of the 5-qubit ring-state, which is genuinely three-colorable and hence can be purified directly only by means of the new protocol. To compare BEPP and MEPP strategies, we computed the maximal reachable fidelity $F_{\max }$, and the minimum required fidelity $F_{\min } . F_{\max }$ is the maximum value of fidelity to which a state of fidelity $F>F_{\min }$ can be brought using the purification protocol. Note that $F_{\max }$ is the same in a communication and in a static scenario. This comes from the fact that the maximal reachable fidelity does not depend on the initial state. That is, if the initial state is distillable, it is always possible to go to a state of fidelity $F_{\max }$.

\section{Communication scenario}

Let us describe the MEPP and the BEPP strategies in the communication scenario. The initial states are different in both strategies which renders the comparison non-obvious. We use the local noise equivalent (LNE), which is, for a state of fidelity $F$, the level of local depolarizing noise defined as in Eq. (25), which has to be applied to the perfect state to obtain fidelity $F$.

In the MEPP strategy, the setting is the following: 5 parties A,B,C,D, and E are connected by depolarizing channels; the party A creates states locally and distributes them to the four other parties. To use direct purification, in addition to the 5-qubit ring states, the party A needs to distribute 5-qubit cluster-states in three different ways, allowing the purification with respect to the three different colors. The purification is done in two steps. The parties first purify the three different cluster states up to their maximum reachable fidelity, before using them to purify the ring-states up to $F_{\max }^{M E P P}\left(p_{l}\right)$, where $p_{l}$ gives the amount of local noise. The fact that $F_{\max }^{M E P}\left(p_{l}\right)$ is always reached for a state with $F>$ $F_{\min }^{M E P} P\left(p_{l}\right)$ is then used to compute $F_{\min }^{M E P P}\left(p_{l}\right)$. For a given amount of local noise $p_{l}$, we vary the channel 


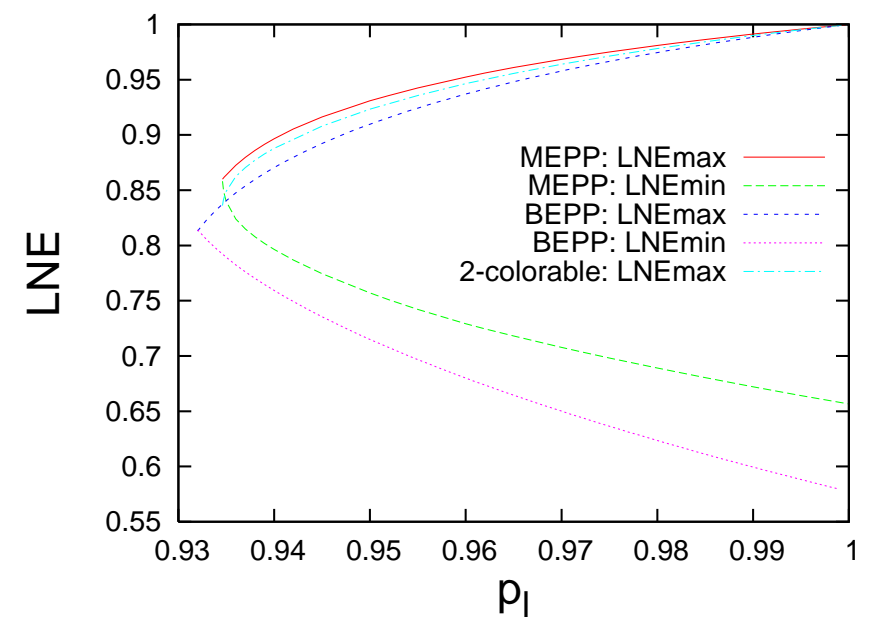

FIG. 5: Local Noise Equivalent (LNE) corresponding to the maximal reachable fidelity and to the minimal required fidelity as function of the amount of local noise $p_{l}$ for the 5qubit ring state in the communication scenario. Given a state of fidelity $F$, the LNE is the level of local depolarizing noise which has to be applied to the perfect state to obtain fidelity $F$. The red solid line and the green dashed line stand for the LNE of the maximal reachable fidelity and the minimal required fidelity respectively for the MEPP strategy. The same values are plotted for the BEPP strategy. The blue small dashed line is the LNE of a ring state teleported using $4 \mathrm{pu}-$ rified pairs and the pink dotted line is th LNE corresponding to the minimal required fidelity of a pair obtained by sending one of its qubits through a depolarizing channel. Not that the maximal reachable fidelity is the same in a communication and a static scenario. We also plotted the maximal reachable fidelity for the alternative strategy where a 5 qubit clusterstate and a Bell pair are purified before being connected. This value is given by the light-blue dotted-dashed line.

noise $q$ to find the threshold value $q_{\min }$ above which the state can be purified up to $F_{\max }^{M E P P}\left(p_{l}\right) . F_{\min }^{M E P P}\left(p_{l}\right)$ is obtained by applying the depolarizing channel with noise parameter $q_{\text {min }}$ to 4 of the qubits of the ring-state.

In the BEPP strategy, the party A creates Bell pairs and distributes them to the other parties. The pairs are purified and then used to teleport locally created 5qubit ring-states. We adopted a conservative scenario where one of the 5 parties creates the ring-states, decreasing by this the number of Bell pairs needed to teleport the states from 5 to 4 . In addition, we assume that the teleportation process itself does not add additional imperfections/noise. Hence the actual value of $F_{\max }^{B E P P}$ is lower than our conservative estimate. To obtain the maximal reachable fidelity of multiparty entangled states $F_{\max }^{B E P P}\left(p_{l}\right)$, we purify the Bell pairs up to $F_{\max }^{B e l l}\left(p_{l}\right)$ and use $N-1$ of them to teleport a locally created ring-state. The results presented in Fig. 5, show that in a communication scenario, the minimum required fidelity as well as the threshold value $p_{l}$, under which no purification is possible is always lower in the BEPP strategy. However, the maximal reachable fidelity is higher in the MEPP

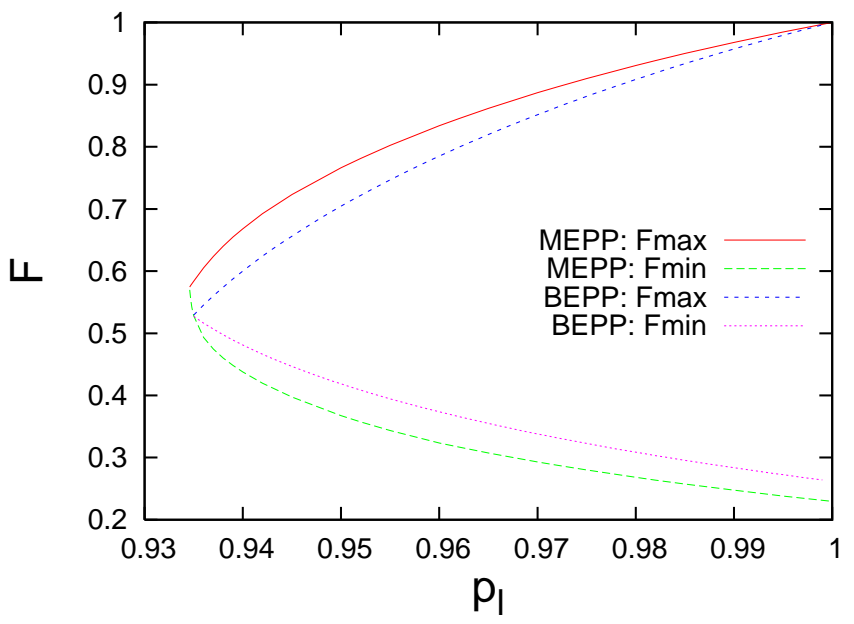

FIG. 6: Maximal reachable fidelity and minimal required fidelity as function of the amount of local noise for the 5-qubit ring state in the static scenario. The red solid line and the green dashed line stand for the maximal reachable fidelity and the minimal required fidelity respectively, for the MEPP strategy, while the blue small dashed line and the pink dotted line stand for the same quantities in the BEPP scenario. The value of $F_{\text {min }}$ plotted for the BEPP scenario is the minimum value of fidelity of the ring state, so that the pairs we extract from the ensemble of rings can be purified.

scenario allowing us to get states of higher fidelity.

We also studied the alternative strategy consisting in distributing two-colorable sub-graph states which are purified and connected at the end. There are two different possibilities depending on the choice of the first color in the construction of the sub-graphs. If one chooses an ensemble of two qubits as first color, one gets a 5-qubit cluster-state and a 2-qubit cluster state (5-2 strategy), while if the first color contains only 1 qubit, one gets a 3-qubits cluster-states plus a 4-qubit one (4-3 strategy). The classification of the different strategies by decreasing value of the maximal reachable fidelity is as follows: direct purification, 5-2 strategy, 4-3 strategy and finally the BEPP strategy. The order is inverted for the value of minimal required fidelity.

\section{Static scenario}

A similar calculation can be done for the static scenario. In this case, one is given an ensemble of distributed 5-qubit ring-states described by $\rho=\prod_{a=1}^{4} \mathcal{E}_{p}^{(a)}|\mathbf{0}\rangle_{G}\langle\mathbf{0}|$. As noted in the previous subsection, the maximal reachable fidelity is the same as in the communication scenario. In the MEPP strategy, the minimum required fidelity is similar in both scenarios. Indeed, the creation of the 3 different cluster states can be done together with a first purification with respect to one of the colors. The behaviour of the protocol in the static scenario therefore doesn't differ much from the one in the communication 
scenario if one begins the purification of the two-colorable graph-state corresponding to graph $g_{j}$, by sub-protocol $\mathcal{P}_{j}$. However, the minimum required fidelity is different in the BEPP strategy. It is defined as the minimum value of fidelity of the ring-state, such that at least one distillable pair can be extracted from it. The results are presented in Fig. 6. The MEPP strategy clearly presents an advantage in terms of fidelity. The minimum required fidelity is lower and the maximal reachable fidelity larger for any value of reliability $p_{l}$.

\section{CONCLUSION}

In this paper, we have proposed multipartite entanglement purification (recurrence and breeding) protocols by which parties can distill arbitrary graph state directly. The work not only gives a complete systematic package for the construction of entanglement purification protocols for graph states, but also clarifies a special role of two-colorable graph states in reading out the parity information of the stabilizer eigenvalues of any graph state. The latter property might open a new avenue to a "patchwork" purification of decohered qubits in resource entan- gled states for quantum information processing. We remark that very recently a similar idea, i.e. the usage of different shapes of graphs, has been utilized by Goyal, McCauley and Raussendorf to obtain purification protocols for two-colorable graph states with improved yield and scaling behavior [20].

We have considered two scenarios, namely (i) the static LOCC scenario and (ii) the communication scenario. Under ideal local operations, we have showed in the static scenario that our protocol gives a higher yield and a wider distillable regime (i.e., a smaller required fidelity for distillability), compared with any bipartite strategy. Also, under noisy local operations, our protocol allows one to distill mixed states up to a higher achievable fidelity in both scenarios.

\section{Acknowledgements}

We thank Simon Anders and Caterina Mora for helpful discussions. This work was supported by the FWF, the European Union (OLAQUI, SCALA, QICS), the ÖAW through project APART (W.D.) and JSPS (A.M.).
[1] C. H. Bennett, G. Brassard, S. Popescu, B. Schumacher, J. A. Smolin, and W. K. Wootters, Phys. Rev. Lett. 76, 722 (1996).

[2] C. H. Bennett, D. P. DiVincenzo, J. A. Smolin, and W. K. Wootters, Phys. Rev. A 54, 3824 (1996).

[3] D. Deutsch, A. Ekert, R. Jozsa, C. Macchiavello, S. Popescu, and A. Sanpera, Phys. Rev. Lett. 77, 2818 (1996).

[4] H. Aschauer and H. J. Briegel, Phys. Rev. Lett. 88, 047902 (2002).

[5] H. J. Briegel, W. Dür, J. I. Cirac, and P. Zoller, Phys. Rev. Lett. 81, 5932 (1998); W. Dür, H. J. Briegel, J. I. Cirac, and P. Zoller, Phys. Rev. A 59, 169 (1999).

[6] M. Murao, M. B. Plenio, S. Popescu, V. Vedral, and . L. Knight, Phys. Rev. A 57, R4075 (1998).

[7] E. N. Maneva and J. A. Smolin, in Quantum Computation and Quantum Information, edited by J. Samuel and J. Lomonaco, Vol. 305 of AMS Contemporary Mathematics (American Mathematical Society, Providence, RI, 2002); quant-ph/0003099.

[8] W. Dür, H. Aschauer, and H. J. Briegel, Phys. Rev. Lett. 91, 107903 (2003).

[9] H. Aschauer, W. Dür, and H. J. Briegel, Phys. Rev. A 71, 012319 (2005).

[10] K. Chen, and H. K. Lo, quant-ph/0404133.

[11] W. Dür, J. Calsamiglia, and H. J. Briegel, Phys. Rev. A 71, 042336 (2005).
[12] W. Dür and H.-J. Briegel, Phys. Rev. Lett. 90, 067901 (2003).

[13] E. Knill, Nature 434, 39 (2005).

[14] M. Hein, W. Dür, J. Eisert, R. Raussendorf, M. Van den Nest, and H. J. Briegel, quant-ph/0602096.

[15] W. Dür, M. Bremner, and H.-J. Briegel, manuscript in preparation.

[16] A. Miyake and H.J. Briegel, Phys. Rev. Lett. 95, 220501 (2005).

[17] M. Horodecki, and P. Horodecki, Phys. Rev. A 59, 4206 (1999); G. Alder, A. Delgado, N. Gisin, and I. Jex, quant-ph/0102035 M.A. Martin-Delgado, and M. Navascues, Eur. Phys. J. D27, 169 (2003); Y.W. Cheong, S.-W. Lee, J. Lee, and H.-W. Lee, quant-ph/0512173

[18] M. Hein, J. Eisert, and H. J. Briegel, Phys. Rev. A 69, 062311 (2004).

[19] C. Kruszynska, S. Anders, W. Dür and H. J. Briegel, Phys. Rev. A 73, 062328 (2006).

[20] K. Goyal, A. McCauley and R. Raussendorf, Phys. Rev. A 74, 032318 (2006).

[21] E. Hostens, J. Dehaene, and B. De Moor, Phys. Rev. A 73, 042316 (2006).

[22] W. Dür, M. Hein, J.I. Cirac, and H. J. Briegel, Phys. Rev. A 72, 052326 (2005).

[23] N. Linden, S. Popescu, B. Schumacher, and M. Westmoreland, quant-ph/9912039. 